\title{
A Novel Technique for Optimizing Panchromatic and Multispectral image fusion using Discrete Wavelet Transform
}

\author{
Ms.V.Ezhilarasi ${ }^{1}$, Dr.V.Rajeswari ${ }^{2}$, Ms.M.Kavitha ${ }^{3}$,Ms.S.Priyadharshini ${ }^{4}$ \\ ${ }^{1}$ Assistant Professor,Information Technology,Karpagam College of Engineering, India. \\ ${ }^{2}$ Associate Professor,Information Technology,Karpagam College of Engineering, India \\ ${ }^{3}$ Assistant ProfessorMCA,Karpagam College of Engineering, India \\ ${ }^{4}$ Assistant Professor,Information Technology,Karpagam College of Engineering, India \\ ezhilarasivijaykumar@gmail.com \\ ${ }^{2}$ rajeswari.vp21@gmail.com \\ 33connecttokavi@gmail.com \\ 4priya.maha.it@gmail.com
}

\begin{abstract}
Image Fusion refers to the process of obtaining a single image by integrating two or more images. The fused image must have complete information which is more useful for human or machine perception. The main aim of image fusion in the satellite images is to improve the information content of the source images, so that the reliability and capability of the image is increased. The vital application of image fusion in remote sensing is to merge the Panchromatic and the Multispectral image. This paper mainly focuses on obtaining a pan sharpened satellite image from Multispectral (MS) and Panchromatic (PAN) images using Discrete Wavelet Transform (DWT) and an iterative algorithm called Tone Mapped image quality index (TMQI-II) is used for optimization. This algorithm mainly improves the structural fidelity and statistical naturalness of the fused image. The earliest fusion schemes perform the fusion on the source images, which often have serious side-effects such as reducing the contrast and spectral distortion. In order to overcome the spectral distortion, Discrete Wavelet Transform is used. IKONOS and Quick Bird satellite images are used to assess the quality of this technique.
\end{abstract}

Keyword--Multispectral (MS), Panchromatic (PAN) images, Discrete Wavelet Transform (DWT), Tone Mapped image quality index (TMQI-II), structural fidelity, statistical naturalness.

\section{INTRODUCTION}

Image Fusion is a technique that integrates multiple images and produces a single image which retains the important information from each of its source image. The images are acquired from different instrument modalities of the same scene (like multi-sensor, multi-focus and multi modal images). Image fusion is widely used in image and signal processing applications such as aerial and satellite imaging, computer vision, robotics, concealed weapon detection and remote sensing. Pan sharpened images are produced by combining the high spectral resolution multispectral image and the high spatial resolution panchromatic image .Panchromatic images are collected in large visual wavelength range and it's rendered in black and white. Multispectral images are obtained from more than one spectral or wavelength interval[11]. Each and every individual image is usually of the same physical location but with various spectral bands[1]. The objective of image fusion algorithms is to make full use of spatial and spectral information in the Panchromatic and Multispectral images respectively, in order to reduce the potential colour distortion and provide clear image. Image fusion improves the quality and increase the application of usage of the data. Image fusion can be classified, depending on whether the images are fused either in the spatial domain or they are transformed into another domain [23].However, the spatial domain image fusion techniques produce spectral distortion while performing the fusion process. In this paper DWT (Discrete wavelet Transform) is used for image fusion using different fusion rules. Further two different types of wavelet are used for fusion process for various fusion rules and it's compared using performance metric. The term resolution is vital in the field of image fusion as it judges the quality of various fused images[14].Image resolution is defined as the smallest measurable detail in visual presentation or it is the amount of detail that an image hold[12]. In satellite image fusion processing the term resolution can be classified into different types.

Spatial or pixel resolution-- Spatial resolution or pixel resolution is called the spacing of pixels in an image and is measured with the set of two positive integer numbers Eg.256 x 245.Higher spatial resolution gives a distinct image with sharp details and subtle colour transitions[15].

Spectral resolution-- It is defined as the smallest resolvable difference in the wavelength. It is describes the sensitivity of the sensor to respond to a particular frequency band. 


\section{EXISTING METHOD}

Over the past several years, image fusion has developed rapidly. It is broadly classified into two domains, the spatial domain and the transform domain. In spatial method, the spatial details can be well injected into the image, but spectral distortion may happen. Some of the techniques are Intensity Hue Saturation(IHS)[6], Principal Component Analysis(PCA)[7],Gram-Schmidt Context Adaptive Sharpening(GSA-CA),Synthetic variable ratio(SVR)[2]. In the recent ten years, the methods based on the Transform framework become well known for their reduced colour distortions, such as wavelets [3], Laplacian pyramids [4],Curvelet Transform[5] but the spatial enhancement is generally not satisfactory, particularly in the case of dissimilarities and aliasing .Most of Earth observation satellites, such as Landsat-7,Spot 5, IKONOS, Quick Bird, GeoEye-1, and WorldView-2 provide both Panchromatic (PAN) images are with high spatial resolution and Multispectral (MS) images are with high spectral resolution. To make full use of the spatial and spectral information, image fusion techniques have been applied in various remote sensing applications. As the representative fusion method of the transform technique, the DWT techniques have clear physical meaning and theoretical basis for better satellite image fusion. However, it was found that the spatial enhancement of the fused image may not be satisfactory[8].

\section{A. Discrete Wavelet Transform (DWT) based fusion.}

Wavelet transform is an effective mathematical tool used in many fields. It decomposes the signal into set of basis function. This basis function is called as wavelets. Wavelets are finite duration oscillatory functions with zero average value. Wavelets can be explained by using two functions i.e. the scaling function $\mathrm{f}(\mathrm{t})$, called as father wavelet and the wavelet function called as mother wavelet .2-D Discrete Wavelet Transformation (DWT) converts the image from the spatial domain to frequency domain[9] .DWT decomposes the image into four components. They are called as low-low, low-high, high-low and high-high frequency components .These four components are referred to as approximation, horizontal, vertical and diagonal coefficients respectively because low-low frequency components contain average information whereas the other components contain directional information due to spatial resolution[10]. Fig 1 depicts the block diagram of Discrete Wavelet Transform.

\section{B. Fusion Rules}

Fusion rules determine how the pixels will be combined. These rules may be application dependent and can be same for all the sub-bands [22]. There are two basic steps to determine the rules. They are as follows (i) compute salient measures corresponding to the individual source transforms.(ii) Decide how to combine the coefficients.

1) Average: It is a well-known fact that regions of images that are in focus tend to be of higher pixel intensity. In this method the resultant fused image is obtained by taking the average intensity of corresponding pixels from both the input image.

$$
K(i, j)=\{X(i, j)+Y(i, j)\} / 2
$$

Where $\mathrm{X}(\mathrm{i}, \mathrm{j})$ and $\mathrm{Y}(\mathrm{i}, \mathrm{j})$ are two input images.

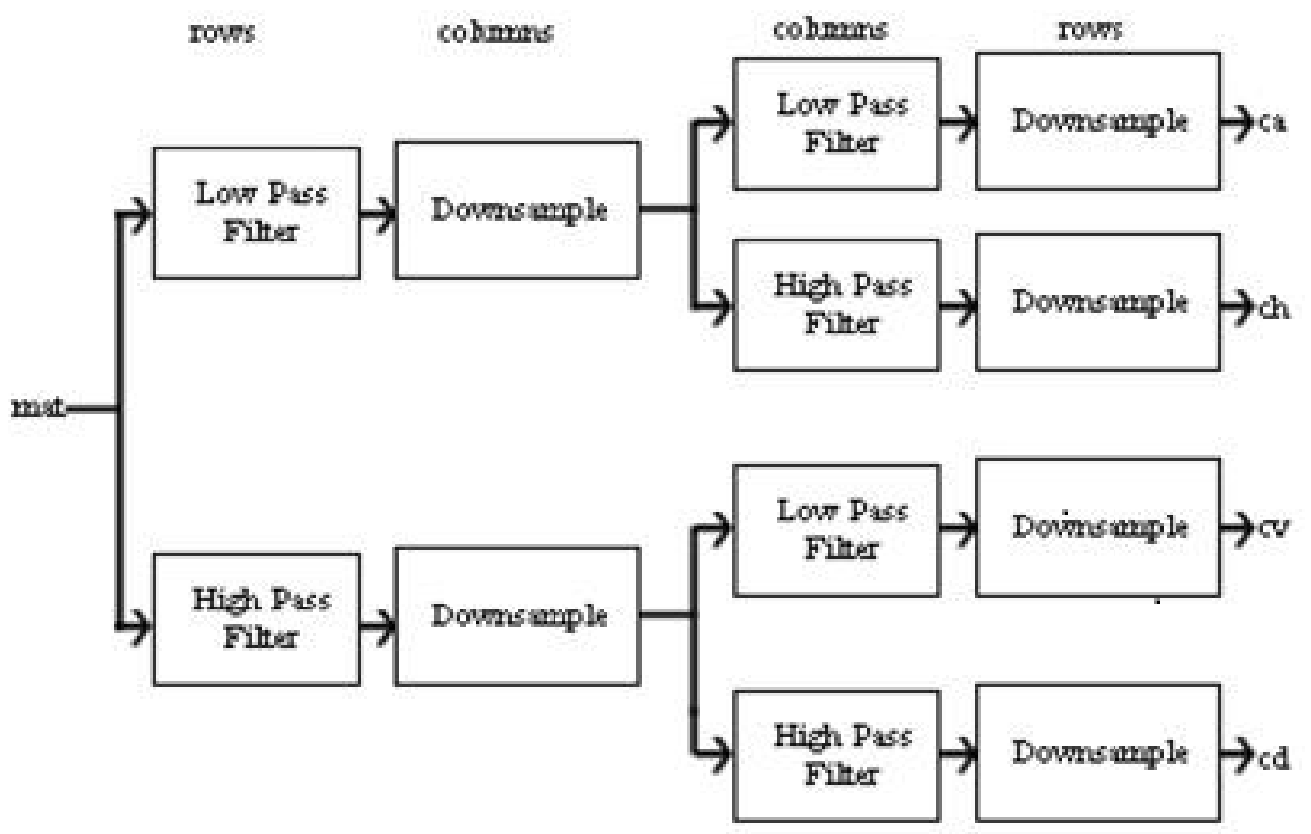

Fig 1. Block diagram of DWT 
2) Simple Maximum: The greater the pixel values the more in focus of the image. This algorithm chooses the in focus regions from each input image by selecting the greatest value for each pixel, there by resulting in highly focused output. The value of the pixel $P(i, j)$ of each image is taken and compared to each other. The greatest pixel value is assigned to the corresponding pixel[15].

$$
\mathrm{P}(\mathrm{i}, \mathrm{j})=\sum_{i=0}^{M} \sum_{j=0}^{n} \max X(I, J) Y(i, j)
$$

Where $X(i, j)$ and $Y(i, j)$ are two input images.

3) Simple Minimum: In this technique, the resultant fused image is obtained by selecting the minimum intensity of corresponding pixels from both the input images[13].

$$
\mathrm{P}(\mathrm{i}, \mathrm{j})=\sum_{i=0}^{M} \sum_{j=0}^{n} \min X(I, J) Y(i, j)
$$

Where $\mathrm{X}(\mathrm{i}, \mathrm{j})$ and $\mathrm{Y}(\mathrm{i}, \mathrm{j})$ are two input images.

\section{III.PROPOSED METHOD}

In DWT based image fusion, first the registered panchromatic and multispectral images are obtained, the corresponding wavelet Daubechies2 (db2) and Daubechies 4(db4) fusion rules are selected and the fusion is done for various decomposition levels. Fig-2 shows the block diagram of DWT based fusion[21].

The wavelet transform can be performed for multiple levels. The next level of decomposition is performed using only the LL image. The result is four sub-images each of size equal to half the LL image size. This process could be continuing to reach the required frequency.We can also increase the levels of transformation if required.

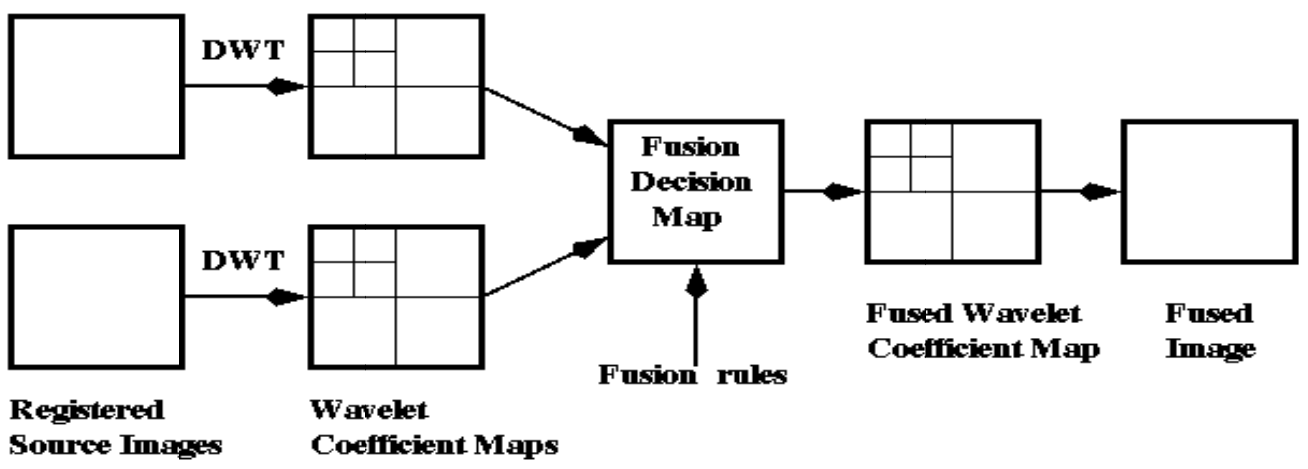

Fig 2. DWT based image fusion

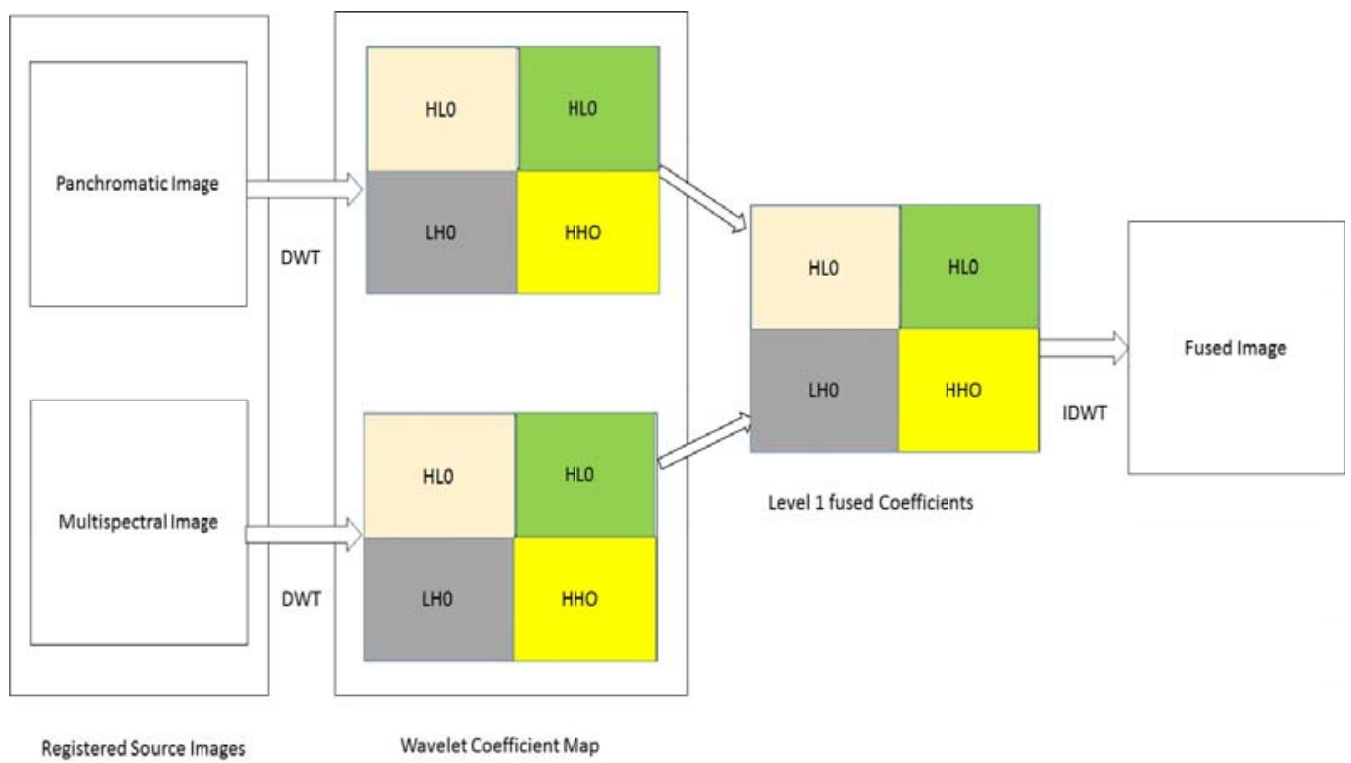

Fig 3.DWT based image fusion for Level 1 decomposition 


\section{Explanation:}

The Registered Source images (Pan and MS images) of the same geographical location are given as the input. Discrete Wavelet Transform is applied for both the registered images to create wavelet decomposoition. Both the input images are decomposed into four sub-bands as LL0,LH0,HL0,HH0. Fuse the different sub-bands by using different fusion rules such as simple Minimum, Simple Maximum, Mean to form the new Wavelet Coefficient Matrix[20]. Reconstructed fused image is obtained by applying Inverse Discrete Wavelet Transform. Fig 3 depicts the DWT based image fusion for level 1 decomposition.

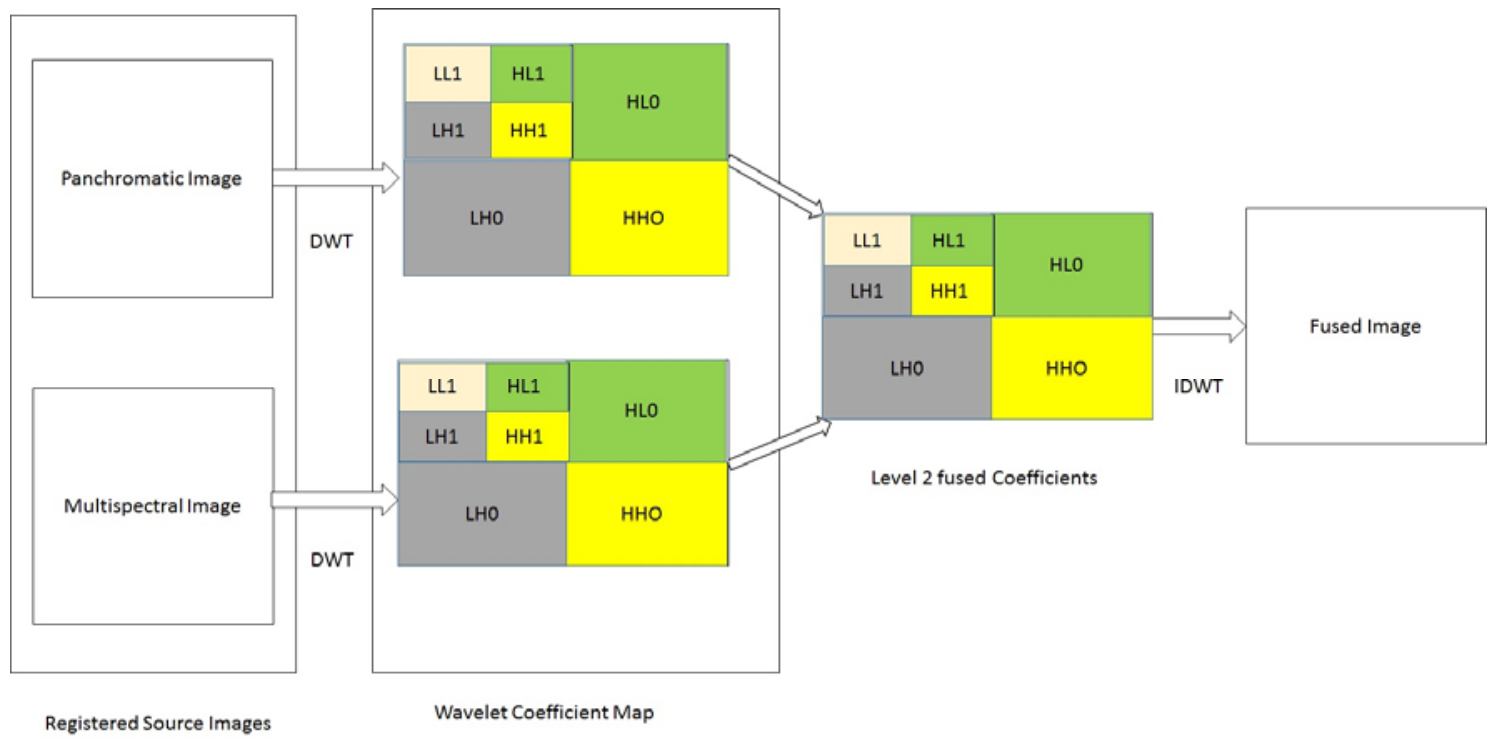

Fig 4 DWT based image fusion for Level 2 decomposition

\section{Explanation:}

Fig 4 DWT based image fusion for level 2 decomposition. The Registered Source images (Pan and MS images) of the same geographical location are given as the input for fusion. Discrete Wavelet Transform is applied for both the registered images to create wavelet decomposoition[19].Both the input images are decomposed into four sub-bands as LL0,LH0,HL0,HH0 for level 1 Decomposition. Again DWT is applied to LL0 Sub-band which is further subdivided into LL1,LH1,HL1,HH1. Fuse the different sub-bands obtained from both the images by using different fusion rules such as simple Minimum, Simple Maximum, Mean to form the new Wavelet Coefficient Matrix. Reconstructed fused image is obtained by applying Inverse Discrete Wavelet Transform.

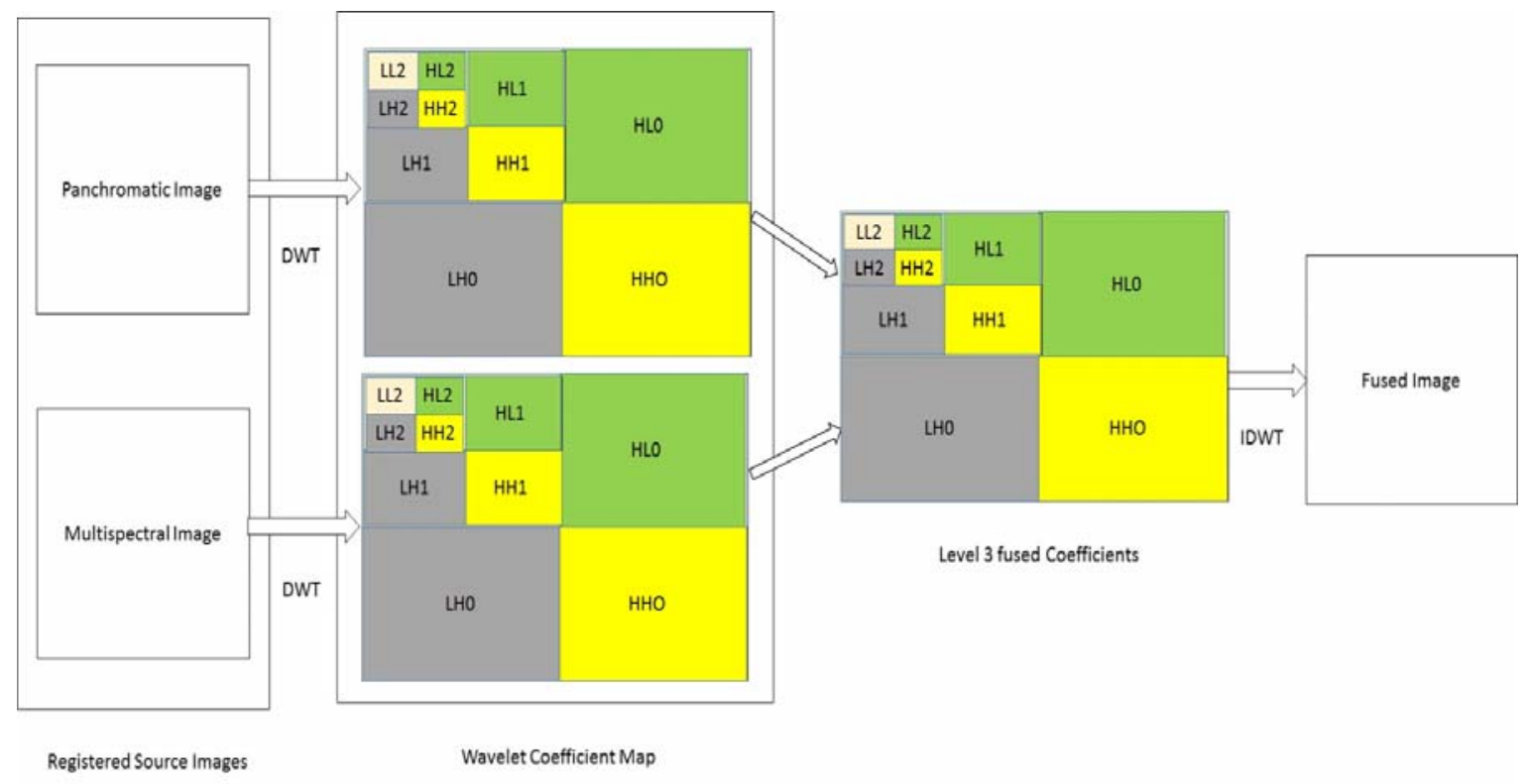

Fig 5 DWT based image fusion for Level 3 decomposition 


\section{Explanation:}

The Registered Source images(Pan and MS images) of the same geographical location is given as the input for fusion. Discrete Wavelet Transform is applied for both the registred images to create wavelet decomposoition.Both the input images are decomposed into four sub-bands as LL0,LH0,HL0,HH0 for level 1 Decomposition.Again DWT is applied to LL0 Sub-band which is further subdivided into LL1,LH1,HL1,HH1 for level 2 decomposition and LL2,LH2,HL2,HH2 for third level of decomposition. Fuse the different sub-bands obtained from both the images by using different fusion rules such as simple Minimum, Simple Maximum,Mean to form the new Wavelet Coefficient Matrix. Reconstructed fused image is obtained by applying Inverse Discrete Wavelet Transform. Fig 5 DWT shows the based image fusion for level 3 decomposition

\section{A.Algorithm for dwt based image fusion}

Input: The registered source images (Pan and MS images) to be fused.

Step 1. Read the two source images, image I and image II to be fused and apply as input for fusion.

Step 2. Implement Discrete Wavelet Transform on both the source images to create wavelet decomposition.

Step 3. Fuse each decomposition level by using different fusion rule like simple average, simple maximum, simple minimum, etc.

Step 4. Concatenation of fused approximations and details gives the new coefficient matrix.

Step 5.Apply Inverse Discrete Wavelet Transform on fused decomposed level, for reconstruction of final fused image and display it.

Step 6.Different wavelet is used now for fusion process and step 2-5 is repeated.

Output: The fused image.

\section{B. Toned mapped optimization algorithm (TMQI-II)}

Tone Mapped image Quality Index (TMQI) is an objective quality assessment algorithm for tone mapped images[18].It consists of two building blocks structural fidelity(S) and naturalness (N).Fidelity measure is based on modified SSIM index and naturalness is based on intensity statistics of images. The structural fidelity of TMQI is computed using a sliding window across the entire image. Consider $\mathrm{x}$ and $\mathrm{y}$ windows in the two images and Slocal is calculated by equation 4 . The overall structural fidelity measure of the image is computed by averaging all local structural fidelity measures by equation 5 .

$$
\begin{gathered}
s_{\text {local }}(x, y)=\frac{2 \sigma_{x \sigma_{y+c 1}}}{\sigma_{x}^{2} \sigma_{y}^{2}+c 1} \frac{\sigma_{x y+c 2}}{\sigma_{x} \sigma_{y+c 2}} \\
s_{\text {local }}(x, y)=\frac{1}{m} \sum_{i=1}^{m} s_{\text {local }}(x i, y j)
\end{gathered}
$$

Where $\sigma x, \sigma y$ and $\sigma x y$ denote the local standard deviations and covariance between the two corresponding images and $x_{i}$ and $y_{j}$ are the $i$-th patches in the image and $M$ is the total number of patches[17]. Statistical Naturalness is used to optimize the brightness and contrast of the image and fidelity is used to optimize the chrominance and also to preserve the local structures of the images. So this algorithm alternatively optimizes both these components and enhances the fused images in each iteration and thereby making the image visually appealing .Naturalness is defined as the product of beta and Gaussian density function. It is calculated by using equation 6.

$$
N=\frac{1}{M} P_{M} P_{D}
$$

$\mathrm{K}$ is the normalisation factor given by $\mathrm{K}=\max \left(P_{M} P_{D}\right)$

So this method is applied to both the fused images which are obtained by using two wavelets [16]. Both the components are improved in each of the iteration process so as to get the good quality image.

\section{Iterative process in steps}

Step 1. Read input images (MS and fused output image).

Step 2. Extract luminance component in both the images.

Step 3. Set the default parameters before iteration.

Step 4. Compute statics like mean \& standard deviation for the images.

Step 5. Set the tolerance value for structural fidelity \& naturalness of the tone mapped image.

Step 6. Structural fidelity and naturalness are calculated and updated for each iteration.

Step 7. Statistical analysis on each iteration.

Step 8 . Find the mean and standard deviation values to the optimized image then compare it with the values obtained from the input image before optimization. 


\section{Implementation and Discussion:}

The Quick Bird satellite image is used in this paper. Each image is with $256 \times 256$ pixels. The two input image Pan and MS is shown in Fig 6. The images are fused using DWT by using various fusion rules for two levels of decomposition using two different types of wavelet. The fused output images without optimization are depicted.
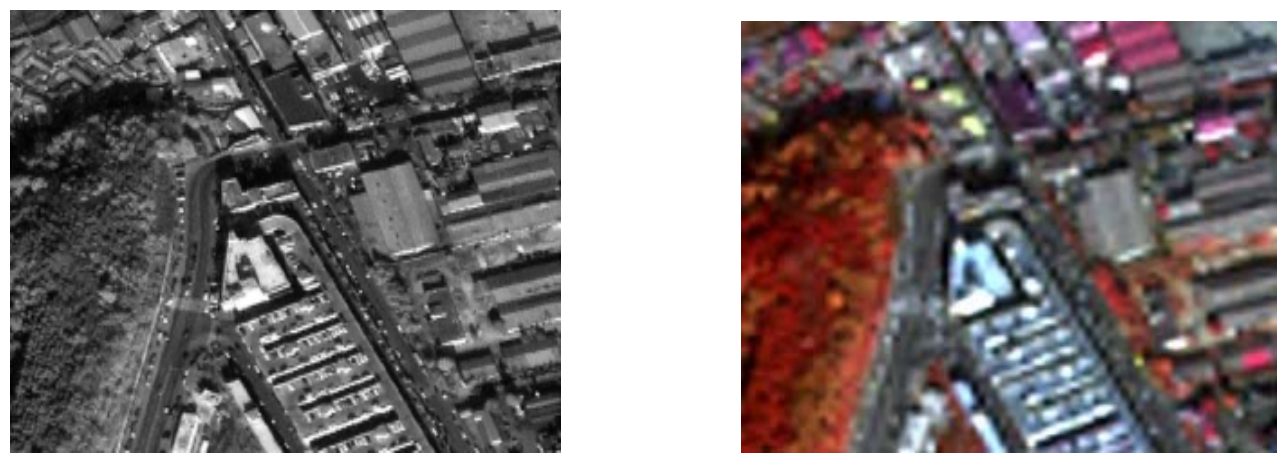

Fig 6 Registered Pan and MS input images

Table 1:Case 1- Level 1 Fused Images without Optimization

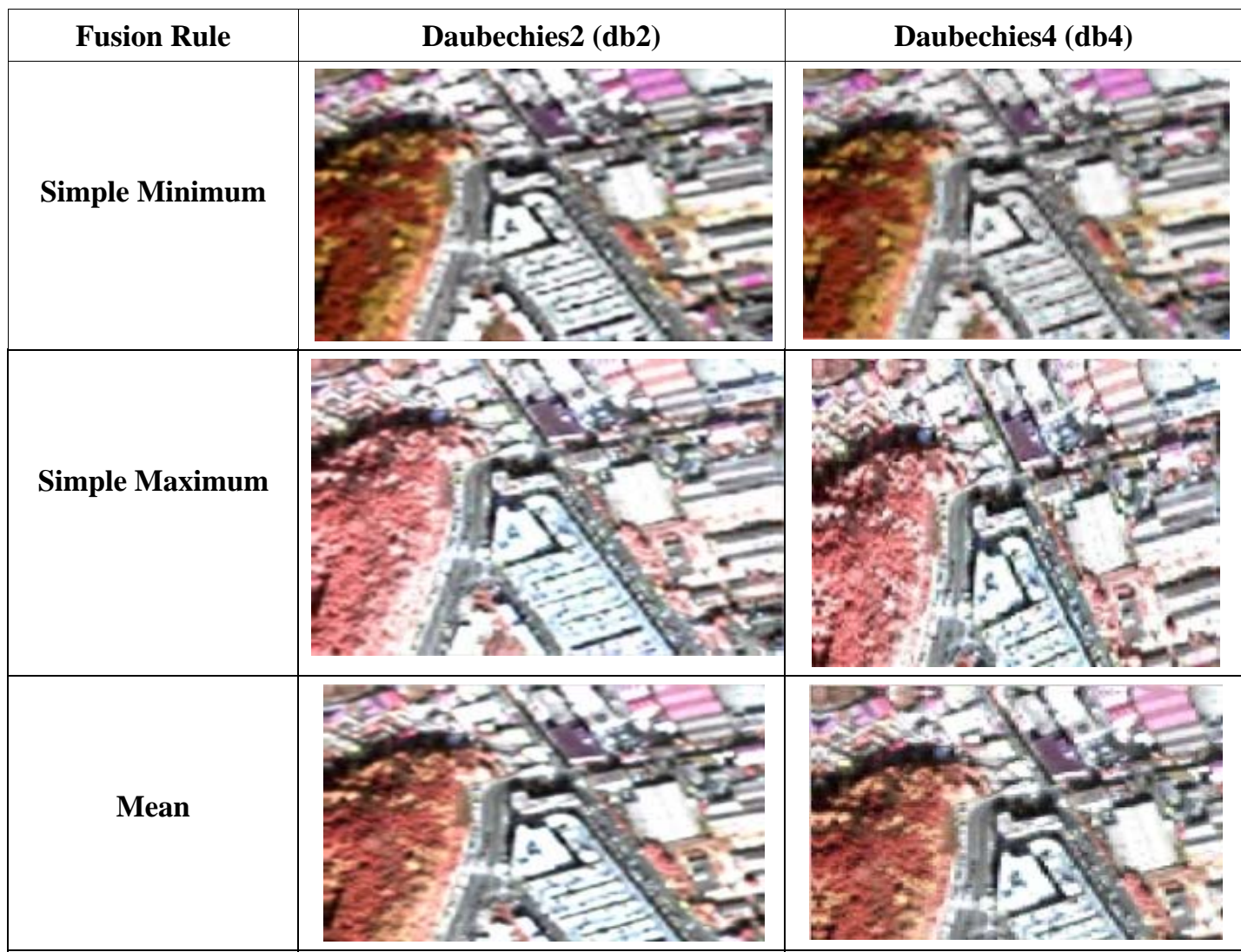

\section{Experimental Results:}

Performance Metric Values for PSNR and SSIM

\begin{tabular}{|c|c|}
\hline Metric & Formulae \\
\hline PSNR & PSNR $=$ 10. Log $10 \frac{M A X_{I}^{2}}{M S E}$ \\
\hline SSIM & $\operatorname{SSIM}(x, y)=\frac{\left(2 \mu_{x} \mu_{y}+c_{1}\right)\left(2 \sigma_{x y}+c_{2}\right)}{\left(\mu_{x}^{2}+\mu_{y}^{2}+c_{1}\right)\left(\sigma_{x}^{2}+\sigma_{y}^{2}+c_{2}\right)}$ \\
\hline
\end{tabular}


Table 2 Metric Values for level 1 of fusion

\begin{tabular}{|c|c|c|c|c|}
\hline \multirow{2}{*}{ FUSION RULE } & \multicolumn{2}{|c|}{ DAUBECHIES2 (DB2) } & \multicolumn{2}{c|}{ DAUBECHIES4 (DB4) } \\
\cline { 2 - 5 } & PSNR & SSIM & PSNR & SSIM \\
\hline Simple Minimum & 23.1497 & 0.9512 & 49.5236 & 0.9612 \\
\hline Simple Maximum & 24.8852 & 0.9642 & 51.2236 & 0.9712 \\
\hline Mean & 22.8542 & 0.9412 & 50.4256 & 0.9563 \\
\hline
\end{tabular}

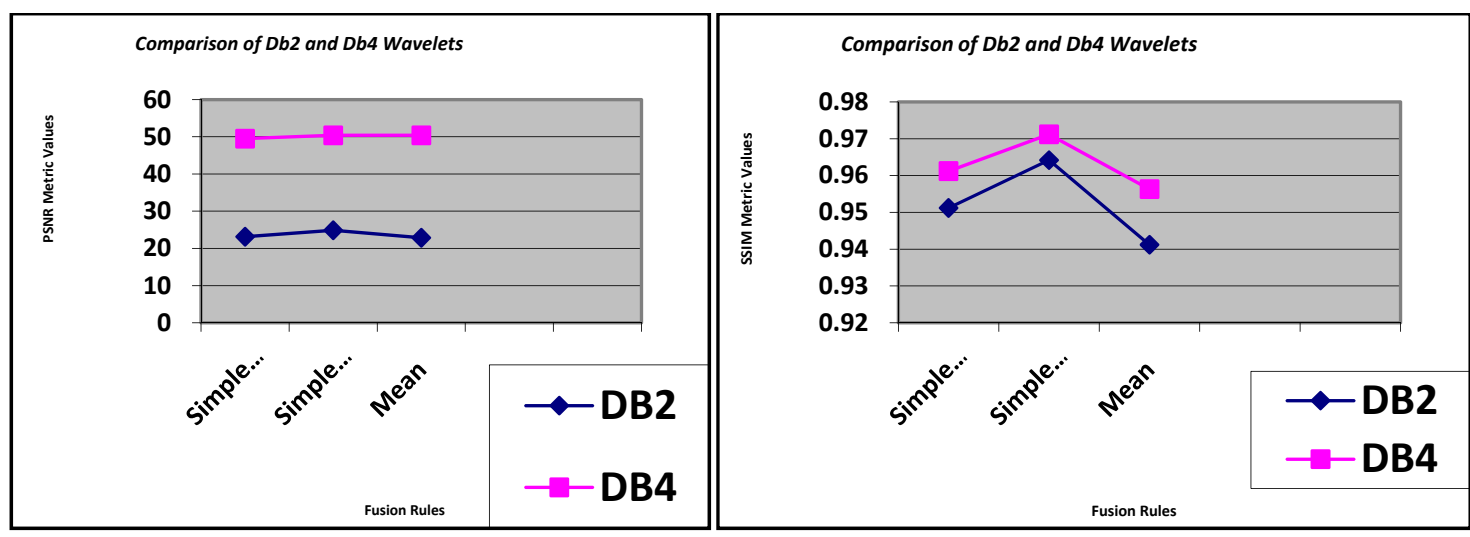

Fig 7 Graphical Representation PSNR Metric and SSIM Metric Values for Level 1 Fusion

Fig. 7 depicts the comparison of metric values of the fused images formed by both wavelets for level 1 fusions. It is notified that the metric values are increasing for Simple Maximum fusion rule for $\mathrm{db} 4$ wavelet.

Table 2 Case 2- Level 2 Fused Images without Optimization

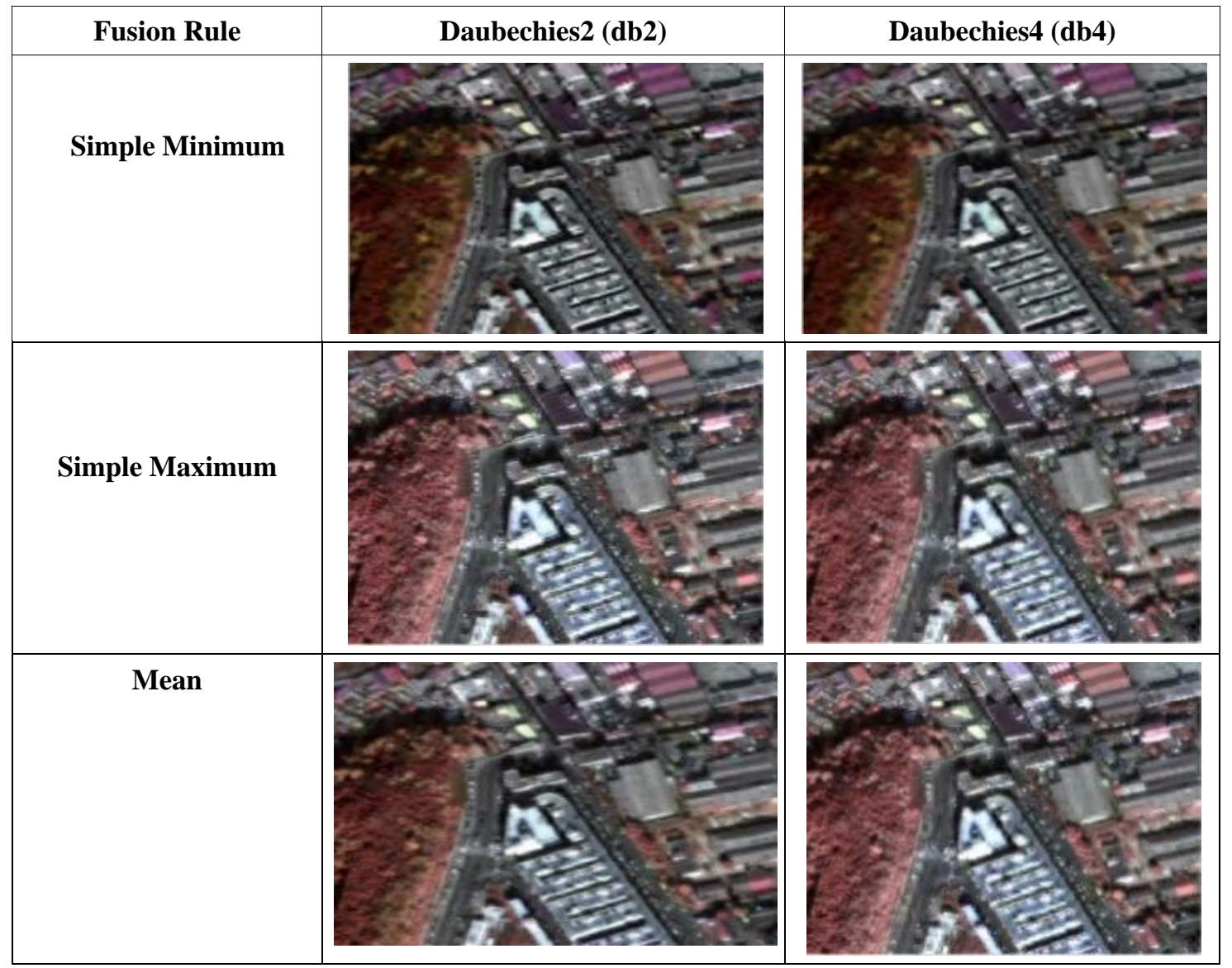




\section{Experimental Results:}

Table 4 Metric Values for level 2 fusion

\begin{tabular}{|c|c|c|c|c|}
\hline \multirow{2}{*}{ FUSION RULE } & \multicolumn{2}{|c|}{ DAUBECHIES2 (DB2) } & \multicolumn{2}{c|}{ DAUBECHIES4 (DB4) } \\
\cline { 2 - 5 } & PSNR & SSIM & PSNR & SSIM \\
\hline Simple Minimum & 25.2899 & 0.9612 & 49.9998 & 0.9714 \\
\hline Simple Maximum & 26.1245 & 0.9745 & 52.4265 & 0.9845 \\
\hline Mean & 24.5246 & 0.9542 & 51.4265 & 0.9612 \\
\hline
\end{tabular}
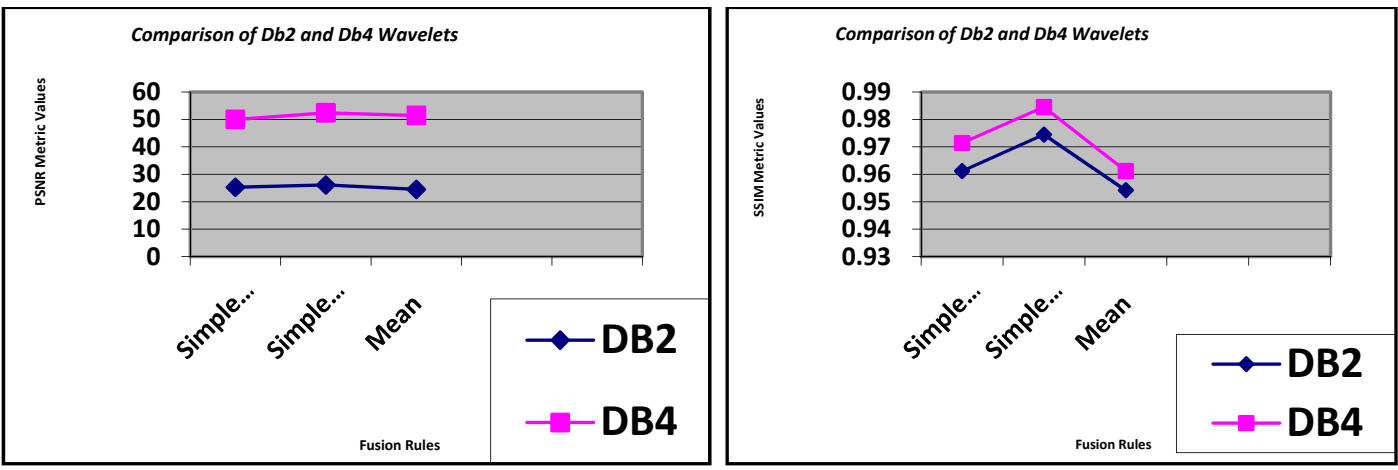

Fig 8 Graphical Representation PSNR Metric and SSIM Metric Values for Level 2

Fig. 8 depicts the comparison of metric values of the fused images formed by both wavelets for level 2 fusions. It is notified that the metric values are increasing for Simple Maximum fusion rule for db4 wavelet.

Table 5 Case 3 -Level 1 Fused Images With Optimization

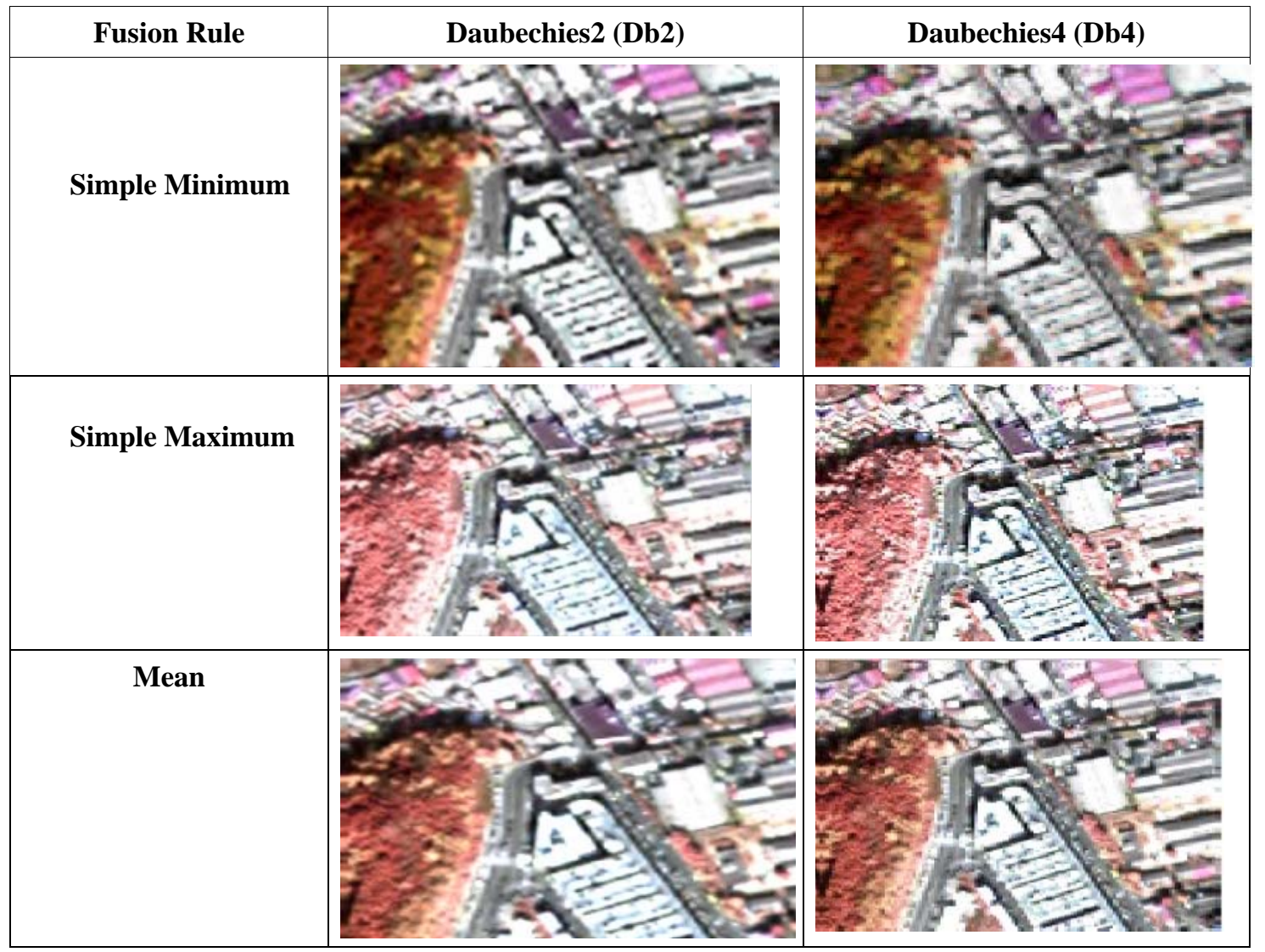




\section{Experimental Results :}

Table 6 Metric Values for level 1 with optimization

\begin{tabular}{|c|c|c|c|c|}
\hline \multirow{2}{*}{ FUSION RULE } & \multicolumn{2}{|c|}{ DAUBECHIES2 (DB2) } & \multicolumn{2}{c|}{ DAUBECHIES4 (DB4) } \\
\cline { 2 - 5 } & PSNR & SSIM & PSNR & SSIM \\
\hline Simple Minimum & 24.1546 & 0.9614 & 51.4123 & 0.9889 \\
\hline Simple Maximum & 25.8965 & 0.9689 & 52.1123 & 0.9814 \\
\hline Mean & 24.5639 & 0.9563 & 50.8962 & 0.9715 \\
\hline
\end{tabular}
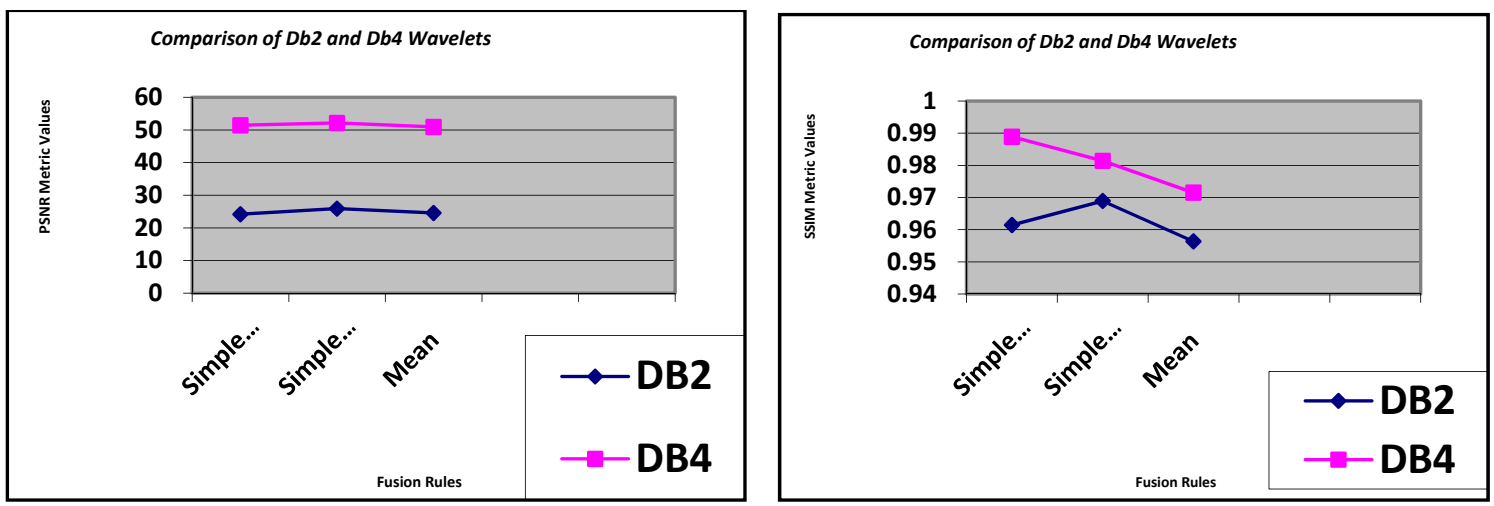

Fig 9.Graphical Representation PSNR Metric and SSIM Metric for Level 1 With Optimization

Table 7 Comparison Of Fused Images With And Without Optimization Based On Fusion Rules: LEVEL 1 Comparison Result FOR PSNR Metrics

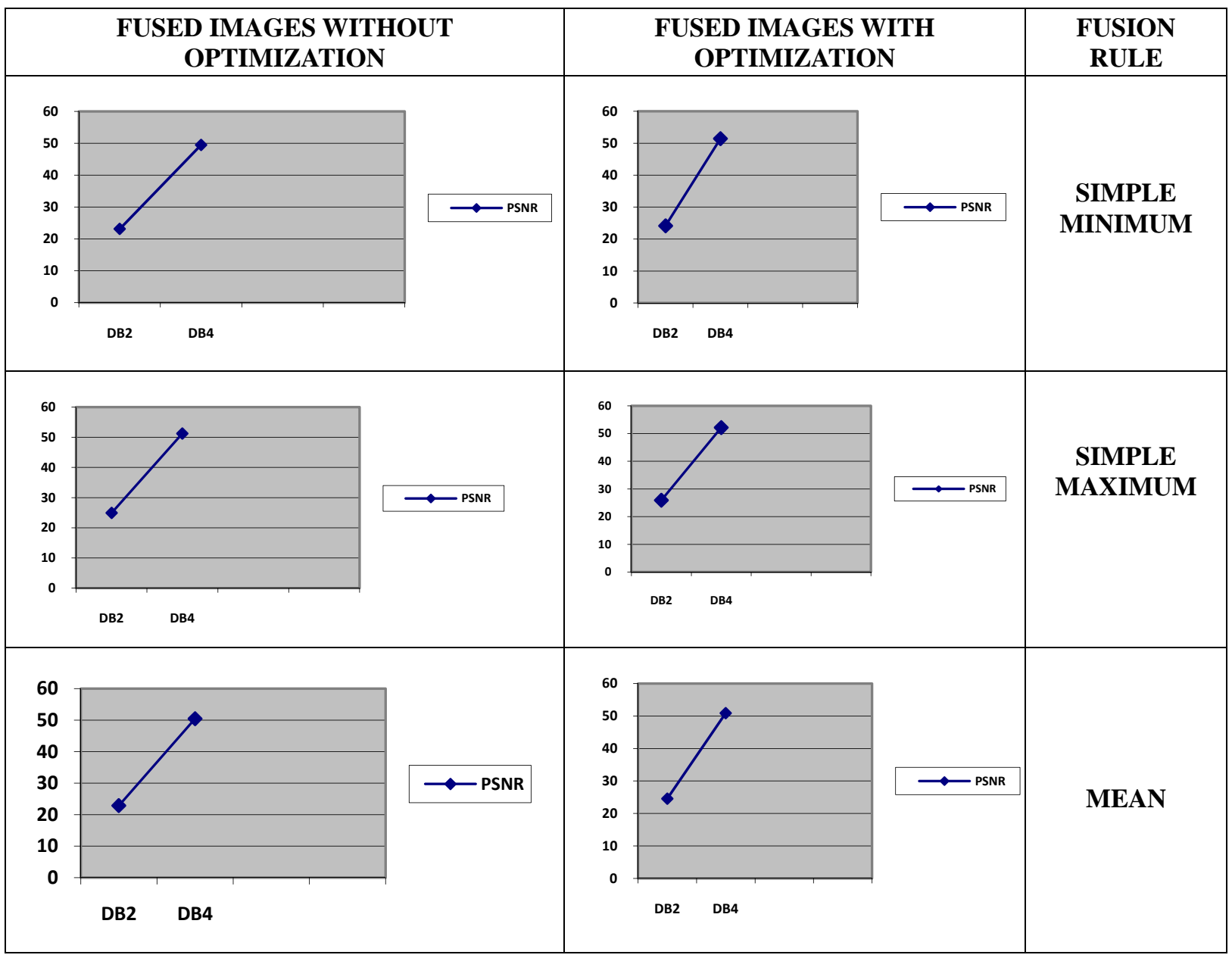




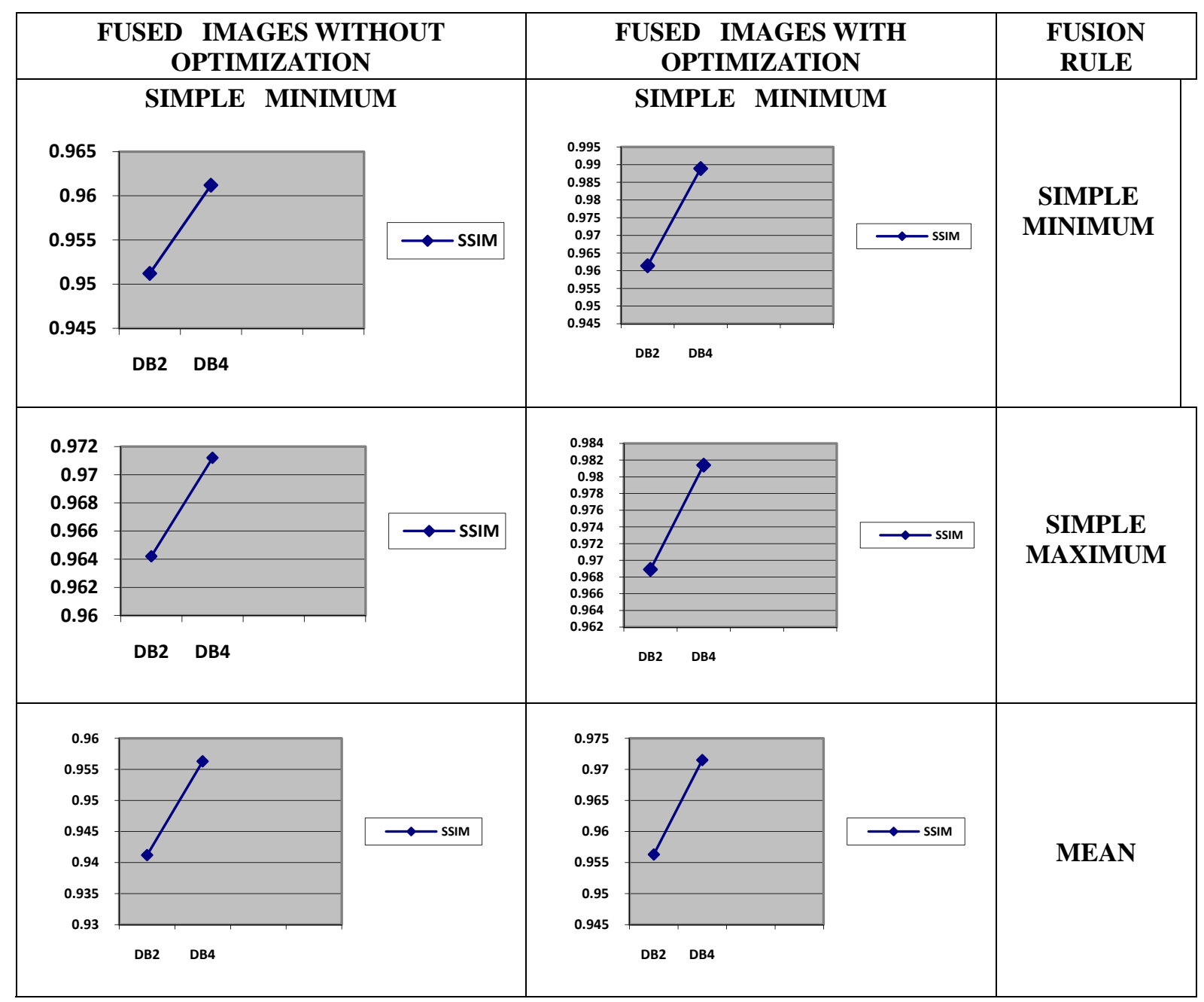


Table 9 Case 4-Level 2 Fused Images Without Optimization

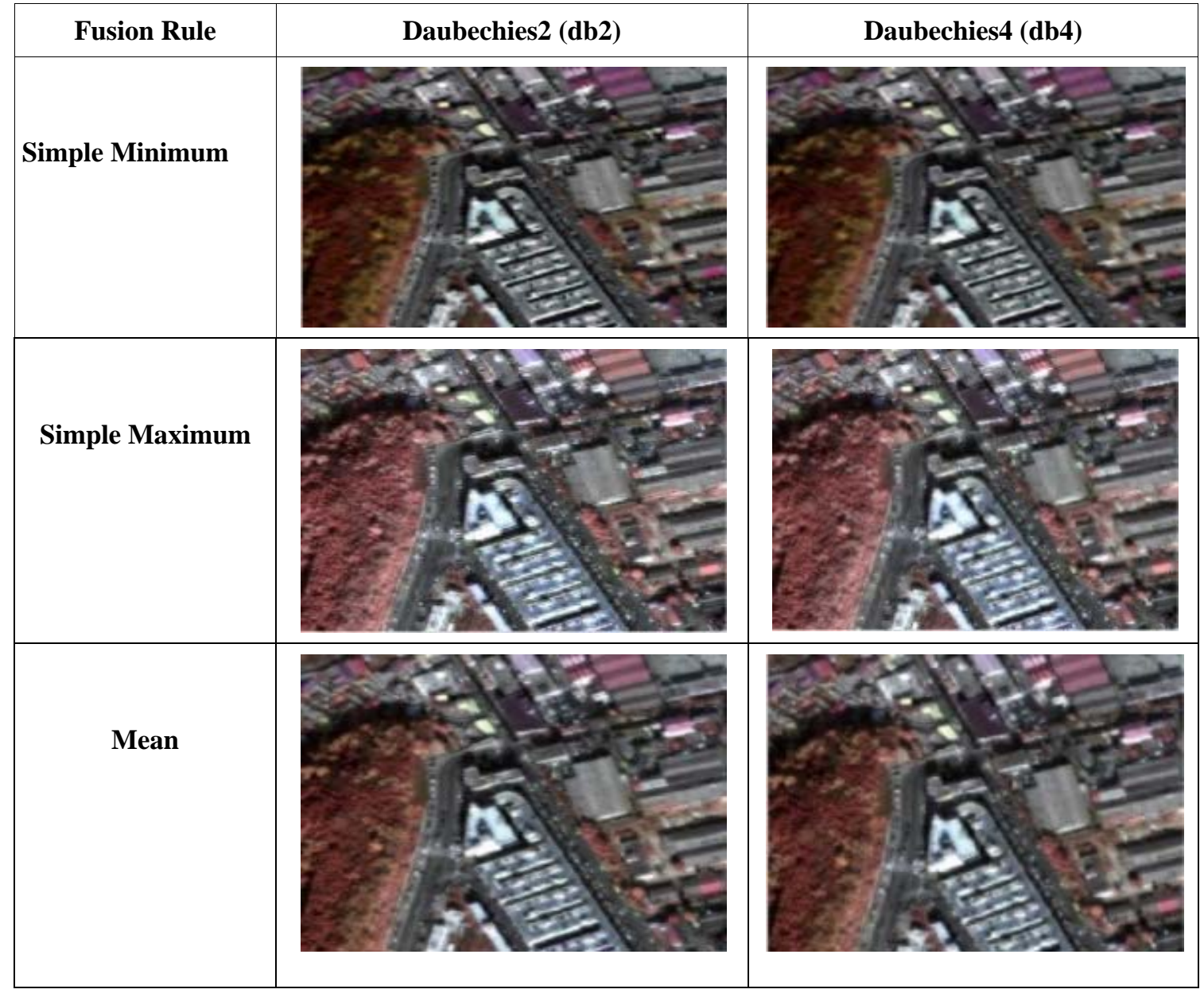

Experimental Results :

Table 10 Metric Values for level 2 with optimization

\begin{tabular}{|c|c|c|c|c|}
\hline \multirow{2}{*}{ FUSION RULE } & \multicolumn{2}{|c|}{ DAUBECHIES2 (DB2) } & \multicolumn{2}{c|}{ DAUBECHIES4 (DB4) } \\
\cline { 2 - 5 } & PSNR & SSIM & PSNR & SSIM \\
\hline Simple Minimum & 26.8965 & 0.9712 & 52.4126 & 0.9845 \\
\hline Simple Maximum & 27.8563 & 0.9841 & 54.1263 & 0.9994 \\
\hline Mean & 25.4125 & 0.9652 & 52.1411 & 0.9899 \\
\hline
\end{tabular}
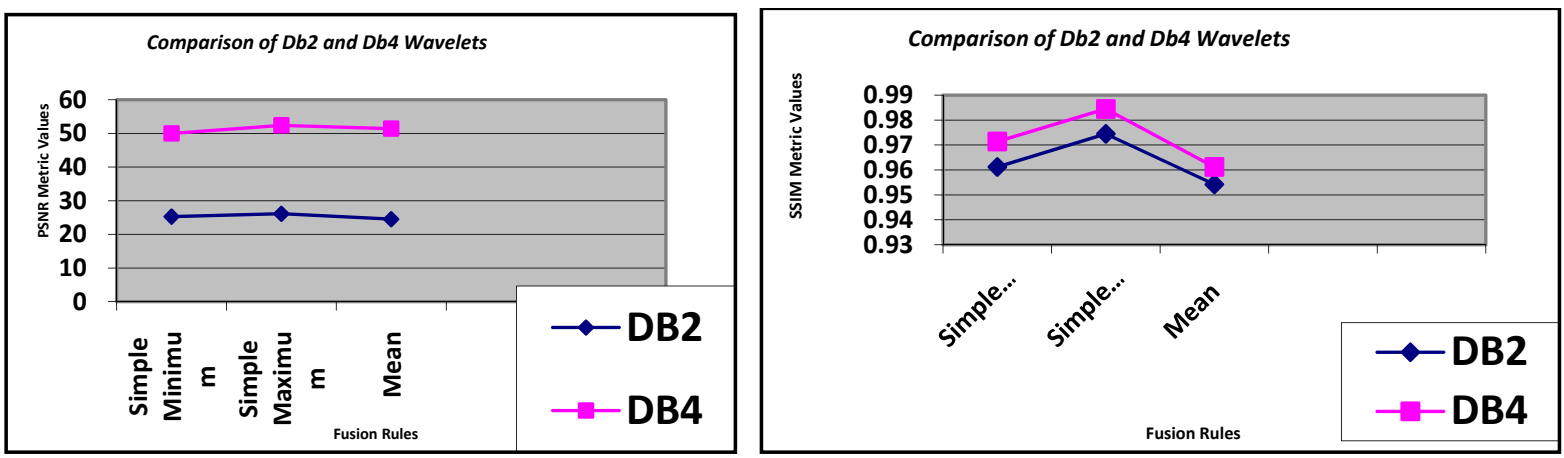

Fig 10. Graphical Representation PSNR Metric and SSIM Metric Values For Level 2 With optimization

Fig. 10 depicts the comparison of metric values of the fused images formed by both wavelets for level 2 fusion. It is notified that the metric values are increasing for Simple Maximum fusion rule for db4 wavelet . 
Table 11 FUSED IMAGES WITH AND WITHOUT OPTIMIZATION BASED ON FUSION RULE FOR LEVEL 2 RESULT FOR PSNR METRICS

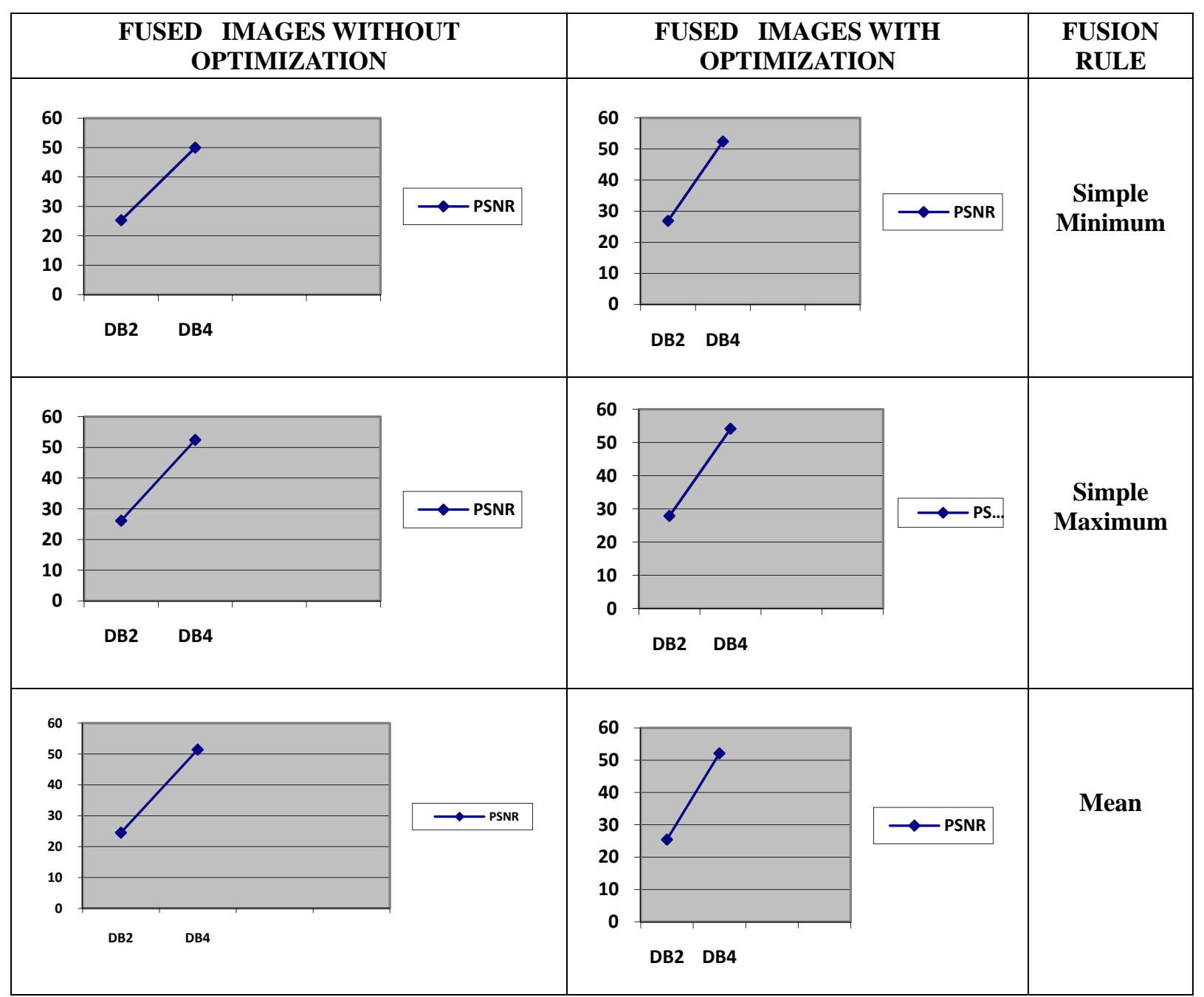


Table 12 Level 2 Result for SSIM Metrics

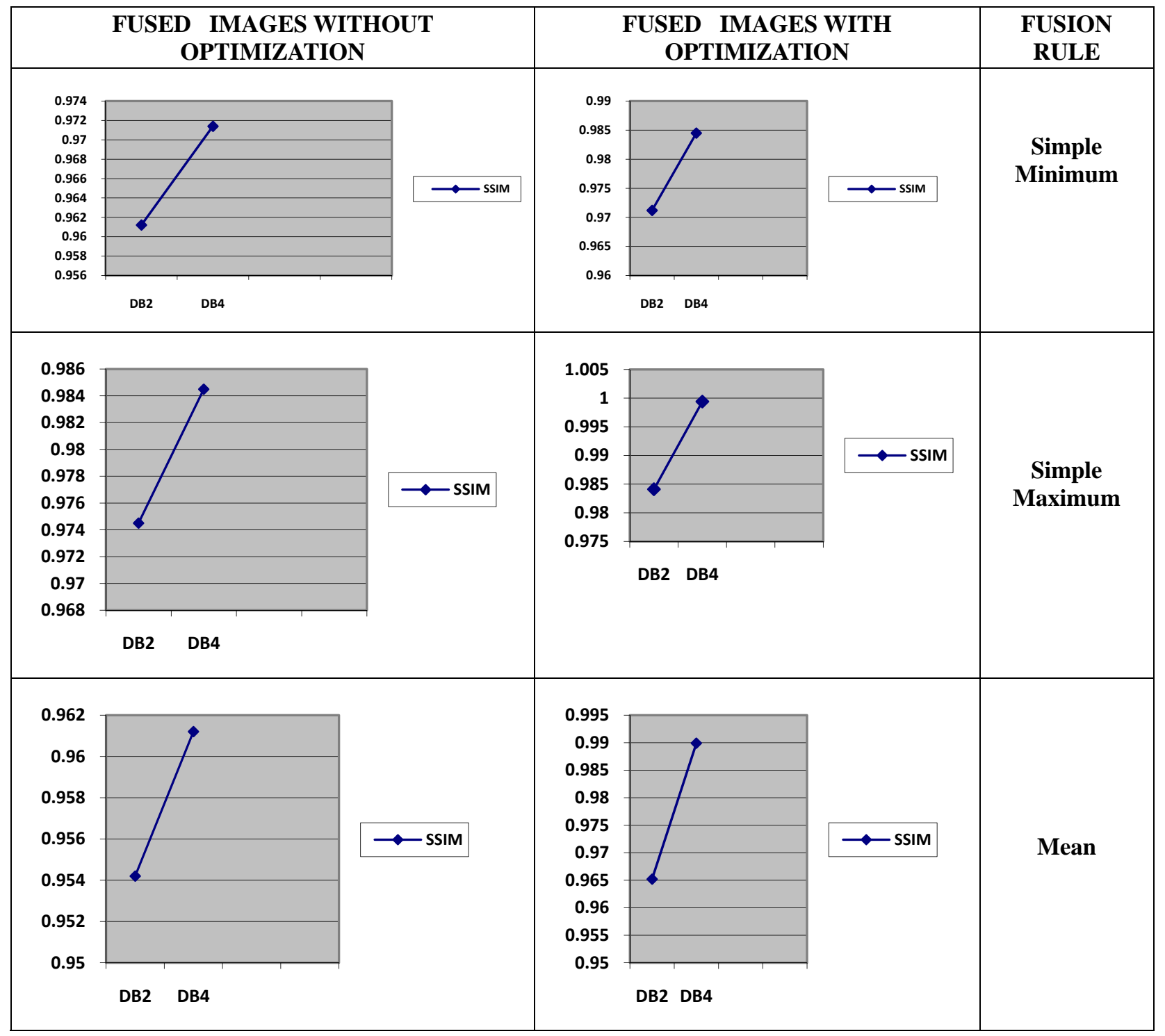

Table 13 Overall Comparison Metric Tables With And Without Optimization

\begin{tabular}{|c|c|c|c|c|c|c|c|c|c|c|}
\hline \multicolumn{11}{|c|}{ Performance Comparison of the Metric Values } \\
\hline \multicolumn{6}{|c|}{ Fused Images without Optimization } & \multicolumn{5}{|c|}{ Fused Images with Optimization } \\
\hline & & \multicolumn{2}{|c|}{ DB2 } & \multicolumn{2}{|c|}{ DB4 } & & \multicolumn{2}{|c|}{ DB2 } & \multicolumn{2}{|c|}{ DB4 } \\
\hline $\begin{array}{l}\text { Levels } \\
\text { of } \\
\text { Fusion }\end{array}$ & $\begin{array}{l}\text { Fusion } \\
\text { Rules }\end{array}$ & PSNR & SSM & PSNR & SSM & & PSNR & SSM & PSNR & SSM \\
\hline \multirow{3}{*}{$\begin{array}{l}\text { LEVEL } \\
\quad 1\end{array}$} & $\begin{array}{c}\text { Simple } \\
\text { Minimum }\end{array}$ & 23.1497 & 0.9512 & 49.5236 & 0.9612 & $\begin{array}{c}\text { Simple } \\
\text { Minimum }\end{array}$ & 24.1546 & 0.9614 & 51.4123 & 0.9889 \\
\hline & $\begin{array}{c}\text { Simple } \\
\text { Maximum }\end{array}$ & 24.8852 & 0.9642 & 51.2236 & 0.9712 & $\begin{array}{c}\text { Simple } \\
\text { Maximum }\end{array}$ & 25.8965 & 0.9689 & 52.1123 & 0.9814 \\
\hline & Mean & 22.8542 & 0.9412 & 50.4256 & 0.9563 & Mean & 24.5639 & 0.9563 & 50.8962 & 0.9715 \\
\hline \multirow{3}{*}{$\begin{array}{c}\text { LEVEL } \\
2\end{array}$} & $\begin{array}{c}\text { Simple } \\
\text { Minimum }\end{array}$ & 25.2899 & 0.9612 & 49.9998 & 0.9714 & $\begin{array}{c}\text { Simple } \\
\text { Minimum }\end{array}$ & 26.8965 & 0.9712 & 52.4126 & 0.9845 \\
\hline & $\begin{array}{c}\text { Simple } \\
\text { Maximum }\end{array}$ & 26.1245 & 0.9745 & 52.4265 & 0.9845 & $\begin{array}{c}\text { Simple } \\
\text { Maximum }\end{array}$ & 27.8563 & 0.9841 & 54.1263 & 0.9994 \\
\hline & Mean & 24.5246 & 0.9542 & 51.4265 & 0.9612 & Mean & 25.4125 & 0.9652 & 52.1411 & 0.9899 \\
\hline
\end{tabular}




\section{Conclusion}

This paper provides the fusion of Panchromatic and Multispectral images using discrete wavelet transform were the wavelet used is daubechies2 and daubechies4 for two levels of decomposition. The images are fused by using three different types of fusion rule (Average, Simple Maximum, and Simple Minimum). The fused images are then optimized by using TMQI-II Algorithm. It is seen that the good quality image is obtained. The fused images are quantified by using the performance metric PSNR and SSIM. It is notified that both the metric value is increased when the images are fused by using the fusion rule absolute maximum and also as we use higher order wavelet the metric values are also notable increased.

\section{REFERENCES}

[1] Qian Zhang,Zhigua Cao,Zhongwen Hu,Yonghong Jia, Joint Image Registration And Fusion Of Panchromatic And Multispectral images , IEEE Geoscience and Remote Sensing Letters, Vol. 12, NO.3, Mar2015.

[2] Huixian Wang, Wanshou Jiang, Chengqiang Lei, Shanlan Qin, and Jiaolong Wang, A Robust Image Fusion Method Based on local spectral and spatial correlation, IEEE Geoscience and Remote Sensing Letters, Vol. 11, NO. 2, Feb 2014

[3] Wavelet for Image Fusion ,Graduate Institute of Communication Engineering \& Department of Electrical Engineering, National Taiwan University

[4] Wencheng Wang,Faliang Chang, A Multifocus Image fusion method based on Laplacian Pyramid, Journal of Computers, Vol. 6, No. 12, December 2011

[5] Myungjin Choi, Rae Young Kim, Moon-Gyu Kim, The Curvelet Transform for Image Fusion , Divison of Applied Mathematics, KAIST 373-1 Guseong-dong, Yuseong-gu, Daejeon, 305-701, Republic of Korea

[6] Z. Zhou, S. Peng, B. Wang, Z. Hao, and S. Chen, An optimized approach for pansharpening very high resolution multispectral images, IEEE Geosci. Remote Sens. Lett., vol. 9, no. 4, pp. 735-739,Jul. 2012.

[7] B. Aiazzi, S. Baronti, and M. Selva, Improving component substitution pansharpening through multivariate regression of MS + Pan Data, IEEE Trans. Geosci. Remote Sens., vol. 45, no. 10, pp. 3230-3239,Oct. 2007.

[8] Keda Ma,Hojatollah Yeganeh,Kai Zeng,Zhou Wang, High dynamic range image compression by optimizing tone mapped image quality index IEEE Image processing,Vol 24.No 10,oct 2015.

[9] G.Divya,N.L Aravinda A new quality evaluation on tone mapped images IJMER.

[10] E.Reinhard,M.Stark,P.Shirley and J.Ferwerda photographic tone reproduction for digital images ACM Trans.Graph,Vol.21,No.3,pp 267-267,2002.

[11] The Influence of Spectral Wavelength on the quality of Pan sharpened image simulated using Hyper spectral data, ISPRS Annals of the Photogrammetry, Remote Sensing and Spatial Information Sciences, Volume I-7, 2012, XXII ISPRS Congress, 25 August - 01 September 2012, Melbourne, Australia.

[12] L.Wang, X.Cao and J.Chen, ISVR: An Improved Synthetic Variable Ratio Method for image fusion, Geocarto International,Vol.23,No.2,April 2008,155-165.

[13] Comparison of Nine Fusion Techniques for very High Resolution Technique, Konstantinos G.Nikolakopoulos .

[14] V. K. Shettigara, A generalized component substitution technique for spatial enhancement of multispectral images using a higher resolution data set, Photogramm. Eng. Remote Sens., vol. 58, no. 5, pp. 561-567,May 1992.

[15] N.H Kaplan,I.Erer,F.Elibol, Fusion of Multispectral and Panchromatic Images by combining bilateral filter and IHS Transform $20^{\text {th }}$ European sifnal processing conference,august 27,2014.

[16] L. Alparone, S. Baronti, A. Garzelli, and F. Nencini, A global quality measurement of pan-sharpened multispectral imagery, IEEE Geoscience ,Remote Sensing. vol. 1, no. 4, pp. 313-317, Oct. 2004.

[17] L. Wald, Data Fusion: Definitions and Architectures Fusion of Images of Different Spatial Resolutions. Paris, France: Presses de l'Ecole Ecole des Mines de Paris, 2002.

[18] I. Ulusoy and H. Yuruk, New method for the fusion of complementary information from infrared and visual images for object detection, IET Image Process., vol. 5, no. 1, pp. 36-48, Feb. 2011.

[19] T.M. Tu, P.S. Huang, C.L. Huang, C.P. Chang, A fast intensity-hue-saturation fusion technique with spectral adjustement for IKONOS imagery , IEEE Trans Geoscience ,Remote Sensing vol.1, no.4, pp.309-312, (2004).

[20] P.S. Chavez, A.Y. Kwarteng, Extracting spectral contrast in Landsat Thematic Mapper image data using selective principal component analysis , Photogrammetric Engineering and Remote Sensing, vol.55, pp. 339-348, (1989).

[21] R. Fattal, D. Lischinski, and M. Werman. Gradient domain high dynamic range compression. In Proceedings of the 29th Annual Conference on Computer Graphics and Interactive Techniques, SIGGRAPH '02, pages 249-256, 2002.

[22] Image Registration and Wavelet Based Hybrid Image Fusion IOSR Journal of VLSI and Signal Processing (IOSR-JVSP) Volume 4, Issue 2, Ver. I (Mar-Apr. 2014), PP 01-05e-ISSN: 2319 - 4200, p-ISSN No. : 2319 - 4197.

\section{Author Profile}

V.Ezhilarasi is working as Assistant Professor in the department of InformationTechnology, Karpagam College of Engineering, Coimbatore. Her areas of interest are Image Processing, VLSI

Dr.V.Rajeswari is Associate Professor in the department of InformationTechnology, Karpagam College of Engineering, Coimbatore. Her areas of interest are J2EE, Database Management Systems and Computer Networks

Ms.M.Kavitha is working as Assistant Professor in department of MCA , Karpagam College of Engineering. Her areas of interest are Database Management Systems, Mobile Networks.

S.Priyadharshini is working as Assistant Professor in the department of InformationTechnology, Karpagam College of Engineering, Coimbatore. Her areas of interest are Big data, Image Processing, Data structures and algorithms. 